\title{
Entretien avec Hervé Regnauld, ancien président de la section 23 du CNU
}

Hervé Regnauld, Jean Gardin, Marie Morelle et Fabrice Ripoll

\section{(2) OpenEdition \\ Journals}

Édition électronique

URL : http://journals.openedition.org/cdg/1204

DOI : $10.4000 /$ cdg. 1204

ISSN : 2107-7266

Éditeur

UMR 245 - CESSMA

Référence électronique

Hervé Regnauld, Jean Gardin, Marie Morelle et Fabrice Ripoll, «Entretien avec Hervé Regnauld, ancien président de la section 23 du CNU », Carnets de géographes [En ligne], 10 | 2017, mis en ligne le 30 septembre 2017, consulté le 14 septembre 2020. URL : http://journals.openedition.org/cdg/1204

Ce document a été généré automatiquement le 14 septembre 2020.

\section{cc)}

La revue Carnets de géographes est mise à disposition selon les termes de la Licence Creative Commons Attribution - Pas d'Utilisation Commerciale - Pas de Modification 4.0 International. 


\title{
Entretien avec Hervé Regnauld, ancien président de la section 23 du CNU
}

\author{
Hervé Regnauld, Jean Gardin, Marie Morelle et Fabrice Ripoll
}

\section{NOTE DE L'AUTEUR}

En mai 2016, nous avons rencontré Hervé Regnauld, en sa qualité d'ancien président de la section $23 \mathrm{du} \mathrm{CNU}$, afin qu'il nous présente la démarche de cette dernière dans l'évaluation des activités d'enseignement des MCF et professeurs d'université. Nous avons considéré qu'il était intéressant de mener un entretien avec un collègue ayant eu ces responsabilité section mais en n'ayant plus à les assumer : Nous pouvions ainsi parier à la fois sur l'expérience d'un mandat complet, d'un certain recul et d'une parole libérée un an après la fin du mandat.

JG-MM-FR : Est-ce tu peux nous raconter rapidement ton parcours personnel ?

Après avoir obtenu son baccalauréat, j'ai fait une "prépa " puis j'entre à l'École normale supérieure de Saint Cloud en 1977. En fait, j'habitais juste en face et mon équipe de rugby (celle du Lycée de Saint Cloud) battait chaque année celle de l'ENS ! C'est la raison pour laquelle je connaissais son existence mais sans être impressionné... Une fois entré, je devais suivre des cours à l'université en parallèle car nous n'étions que deux étudiants en géographie à l'ENS, ce qui ne permettait pas d'ouvrir des cours. La seule université ouverte à l'heure et la date où nous cherchions à nous inscrire était Paris 4 où il y avait deux cours obligatoires et un en option. Or le seul cours en option qui ne tombait pas le jour du sport était «géographie de la mer ", et le professeur nous a emmenés en campagne océanographique! Voilà pourquoi je suis devenu morphologue littoraliste: parce que c'est le seul cours qui n'avait pas lieu le jeudi! Puis j'obtiens l'agrégation (en 1981), et après mon service militaire, je suis nommé dans un collège de l'Oise, à $15 \mathrm{~km}$ de la première gare... Je débute ma thèse alors que je suis en $5 \mathrm{e}$ année d'ENS puis je l'ai poursuivie pendant 
que j'enseignais dans le secondaire. Je suis aussi chargé de cours à Paris 4 à cette période, et je fais des cours en prépa. À l'époque de ma soutenance, en 1985, il n'y a pas de poste de MCF disponible. Et en 1989, dix postes de MCF s'ouvrent, sur lesquels je postule. Je suis classé plusieurs fois premier et je choisis l'université de Rennes 2, et non Paris 7, ce qui en a surpris plusieurs... Bon je précise que, si j'adore Paris, j'ai à cette époque trois enfants en bas âge et je considère que Paris n'est pas adapté alors qu'à Rennes chacun des enfants allait avoir sa propre chambre. Je deviens professeur en 2002 dans cette même université où j'ai assumé diverses responsabilités (directeur de département, membre du conseil d'administration de l'université puis du PRES Université européenne de Bretagne). Je suis élu à la section 23 du CNU et j'en deviens président de 2011 à 2015.

\section{De l'impossibilité d'évaluer les activités d'enseignement...}

JG-MM-FR : Donc tu as été président de la section 23 du CNU qui a la responsabilité de décider de la «qualification » ou non des candidats aux emplois de maître de conférences et de professeur en géographie, ainsi que de l'attribution d'une partie des promotions et des primes demandées par les titulaires. Les MCF et les professeurs étant non seulement des chercheurs mais aussi des enseignants, pourrais-tu nous dire si vous preniez en compte la partie enseignement de leurs activités dans vos évaluations et décisions, et si oui de quelle manière?

HR : Au CNU, nous étions plusieurs à penser que nous devions tenir compte de l'enseignement. Mais, dans la pratique, on ne juge que des dossiers papier, jamais des individus ; or sur des dossiers papier, n'importe qui peut raconter n'importe quoi en matière d'enseignement. Il nous est arrivé d'évaluer des dossiers dans lesquels les candidats annonçaient une quantité si importante de charges de cours, de responsabilités pédagogiques et d'encadrements divers (masters, thèses, etc.), qu'elle nous semblait physiquement impossible à assurer! Surtout quand s'y ajoutait un grand nombre de communications de colloque, de publications, etc. Il est tout à fait possible qu'il y ait des gens qui fassent remarquablement bien leur travail mais il y a des dossiers qui annoncent 5 directions de thèses, 15 ou 20 directions de masters 2 et autant de masters 1 par an, ce qui n'est pas pensable, à moins de ne voir chaque étudiant qu'une demi-heure par an... Nous nous sommes donc très vite rendu compte que nous ne pouvions absolument pas prendre au sérieux certaines déclarations faites sur les responsabilités et services d'enseignement. Et comme elles sont invérifiables, nous avons décidé de ne pas les considérer comme le critère déterminant. Et dans un souci d'égalité, pour les promotions en particulier, nous avons décidé de ne juger les dossiers que sur les éléments que nous pouvions vérifier, c'est-à-dire les publications, qu'il s'agisse d'articles, d'ouvrages ou de chapitres d'ouvrage collectif. Il ne s'agissait pas de s'occuper de bibliométrie: nous étions surtout attentifs au fait que les articles soient publiés dans des revues différentes, avec des referees différents, de même pour les livres, qu'ils soient édités chez des éditeurs différents, etc., car cela montrait que le candidat ou la candidate ne jouait pas uniquement dans son cercle fermé. 
JG-MM-FR : Autrement dit, le CNU, contraint et forcé, reconduit le fait que l'enseignement reste une boite noire finalement.

HR : Disons que le CNU constate que l'enseignement est une activité à propos de laquelle on doit croire sur parole ce qu'annoncent les candidats étant donné qu'on n'a aucun moyen de le vérifier. Donc c'est clair qu'on aurait aimé être capable de prendre effectivement en compte la réalité pédagogique de la charge d'enseignement... mais en fait on ne peut pas. À un moment, nous avions suggéré que les enseignants qui le voulaient mettent leurs cours en ligne sur HAL. C'est parfaitement possible pour les cours de master, pas pour les cours de licence. C'est assez fréquent dans les disciplines scientifiques mais c'est extrêmement rare en géographie. Cela aurait permis de voir les cours proposés, de vérifier que celui qui dit faire, disons $150 \mathrm{~h}$ en master, ne raconte pas 150 fois la même chose... ou ne se contente pas de lire ses propres articles, et qu'il fabrique véritablement un cours... Il n'est pas question de demander que la mise en ligne soit obligatoire, bien sûr, c'est hors de question. Mais même quand c'est proposé à titre facultatif, on s'aperçoit que quasiment personne ne le fait, donc il n'y a pas moyen de vérifier. Contrairement à d'autres sections du CNU, la section 23 n'exige d'ailleurs pas qu'il y ait une expérience d'enseignement pour qualifier aux fonctions de maître de conférences. Parce qu'il y a beaucoup de doctorants en géographie qui font du terrain, à l'étranger notamment, ce qui les empêche, d'un point de vue purement pratique, d'accumuler les heures d'enseignement.

JG-MM-FR : En tant que candidat aux postes de MCF, on a pourtant entendu dire qu'il fallait absolument avoir une expérience d'enseignement et qu'il fallait bien indiquer le nombre d'heures effectuées sur le CV car plus il y en avait et meilleures étaient les chances d'être qualifié puis recruté. Et depuis, en tant que titulaire, ce que l'on entend dire des collègues dans les discussions informelles comme dans les comités de sélection, confirme que la quantité d'heures vaut expérience, et que la question de savoir ce que les personnes faisaient vraiment, si elles concevaient ou non le cours, et quelle était la qualité ou la pertinence de ce qui était fait, était une question qui restait sous le tapis parce qu'une fois énoncée elle fiche tout par terre! Mais effectivement, si l'on considère que l'expérience d'enseignement n'est pas éliminatoire, comment évaluer la capacité à enseigner, comment construire cette capacité ?

HR : Alors la capacité à enseigner en géographie, elle est censée être acquise par le fait de suivre un cursus de géographie. Là où il y avait des discussions, c'est quand des personnes demandaient à être qualifiées en géographie en ayant un cursus dans une autre discipline, par exemple en sociologie, en économie, etc. Il y a $20 \%$ des candidats à peu près qui viennent d'autres disciplines. Donc on regardait dans ces cas là si, bien qu'ils aient suivi une formation en économie ou en sociologie, ils avaient déjà enseigné dans un département ou une UFR de géographie, s'ils avaient déjà participé à un séminaire avec des géographes, co-écrit des articles avec des géographes, ce genre de choses. C'est la raison pour laquelle ces précisions avaient été données sur le site du CNU. Mais encore une fois, si quelqu'un nous dit qu'il a travaillé à plusieurs reprises dans des ateliers transversaux avec des géographes, on n'a absolument aucun moyen de le vérifier. Il peut très bien avoir été invité à un séminaire de master pour parler en tant que sociologue. Ce qui ne serait pas du tout illogique enfin, cela n'a rien de mal. Mais ça n'implique pas pour autant qu'il puisse faire un cours de géographie en première année sur un commentaire de document ou autre exercice basique de ce genre. Comme on n'avait aucun moyen de vérifier, on a fini par considérer que ce n'était pas un argument qui permettait d'exclure quelqu'un. Et puis 
nous avions calculé que nous avions en séance, un temps moyen de sept minutes à consacrer à chaque candidat! Donc nous avions décidé que si un candidat avait une thèse " raisonnablement bonne » (qui pouvait avoir des défauts mais...), qu'il avait un minimum d'enseignements et disons deux publications, on traitait son dossier en 15 secondes, ce qui permettait de garder du temps pour ceux qui posaient des problèmes, qui étaient pour la plupart des candidats étrangers ou qui n'avaient pas le même cursus, etc. Donc, pour ce qui est de la qualification, on considérait qu'il fallait donner sa chance à tout candidat qui avait un minimum de recherche et de pédagogie. Et on se disait que c'était à la commission de spécialistes de décider...

JG-MM-FR : Ce qui ne fait que reporter le problème sur d'autres instances qui n'ont pas beaucoup plus les moyens de vérifier. Dans les « recommandations » mises en lignes par la section 23 sur le site du CNU, les critères pris en compte pour la qualification aux fonctions de professeur comportent l'implication dans les formations professionnelles, « notamment les licences et masters professionnels», avec comme activités "la coordination pédagogique », "le suivi de stage», "l'aide à la recherche d'emploi », «le lien avec les milieux professionnels »'. Cela peut donner l'impression que ceux qui enseignent dans les cours de licence "générale», les TD de première année, les cours de géographie pour historiens, etc., seraient moins valorisés. C'est un peu surprenant. Pourquoi mettre l'accent sur les filières professionnelles, courtes?

HR : Justement, comme il y a de plus en plus de filières professionnelles, qui sont de bonnes formations, le fait qu'un professeur ne fasse pas que des cours en M2 recherche, mais qu'il participe à une licence pro, qui est un équivalent de L3, c'est un signe important de son implication. Il faut dire qu'on avait vu des dossiers de professeurs, pour les promotions, qui se constituaient des emplois du temps quasiment « sur mesure », avec deux cours en M1, l'essentiel en M2, qui plus est dans le master qu'ils avaient initié 㕈 ce qui voulait dire qu'ils avaient 12 étudiants. Un professeur, c'est quelqu'un qui doit mouiller sa chemise : faire des TD pour historiens effectivement, des cours d'orientation pour tout public, des licences professionnelles en horticulture et paysage, etc. C'est aussi important que de faire un M2 sur son propre sujet de recherche...

JG-MM-FR : Toi qui avais participé à des cessions CNU précédentes, en 1992-93, et a donc du recul sur ces questions, est-ce qu'il est possible, sur ce volet enseignement, de distinguer une évolution?

HR : Au début des années 1990, c'était totalement différent. Le CNU ne fonctionnait pas du tout de la même façon pour les professeurs: la section délibérait après les décisions de recrutement des commissions de spécialistes et pouvait casser ces décisions a posteriori. On n'était pas qualifié, on était approuvé une fois qu'on avait été recruté par une commission de spécialiste en local. Donc on ne peut pas comparer le CNU dont je faisais partie au début des années 1990 avec celui d'aujourd'hui, en matière de qualification des professeurs.

JG-MM-FR : Et qu'en est-il de la place de l'agrégation par exemple? Certaines personnes semblent considérer qu'avoir l'agrégation constitue une garantie que l'on est un bon enseignant, et un bon enseignant à vie. II est vrai qu'en géographie, avoir l'agrégation n'a peut-être pas autant été une condition nécessaire pour devenir titulaire qu'en histoire par exemple, mais n'est-ce pas une conception traditionnelle qui est en train d'être remise en question?

HR : C'est un point qui était effectivement débattu au CNU, sur lequel il n'y avait pas d'opinion claire et consensuelle. Moi j'ai juste une opinion personnelle. Je pense que l'agrégation a un intérêt. Déjà cela confère un salaire et un statut. La deuxième chose 
c'est que, pour avoir l'agrégation, il faut travailler les questions au programme qui ne sont pas forcément celles qui passionnent. Cela veut dire qu'il faut au minimum être capable de faire une dissertation pas trop médiocre sur un sujet qui n'est pas forcément sa tasse de thé. Donc ça laisse supposer qu'un agrégé peut préparer un cours à peu près convenable $\mathrm{y}$ compris sur un sujet dont il n'est pas spécialiste. Après ce n'est pas parce que tu es agrégé que tu vas être bon pédagogue... Au moins es-tu capable de brasser des thématiques qui sortent de ton domaine de prédilection. Notez que c'est mon opinion personnelle et non pas l'opinion du CNU. Mais on est un certain nombre au CNU à penser que l'agrégation a au moins cet intérêt-là.

JG-MM-FR: Mais cela peut aussi donner le sentiment qu'on est capable de parler avec autorité sur n'importe quoi...

HR : Non c'est l'inverse. Quelqu'un qui a eu à travailler deux ou trois sujets éloignés de ses thèmes, hors programme, sait très bien qu'il n'est pas capable de parler de n'importe quoi.

\title{
La prépondérance du critère des publications : une mise en concurrence des activités de l'E-C?
}

\begin{abstract}
JG-MM-FR : Ce n'est tout de même pas très satisfaisant comme critère, ne serait-ce que parce que l'absence d'agrégation ne signifie pas incompétence en matière d'enseignement. Mais si, au nom de cette absence de critère satisfaisant et déterminant en matière d'enseignement, le CNU tente de valoriser l'activité de publication et «l'ouverture » à travers la diversité des supports, cela ne risque-t-il pas de pénaliser celui ou celle qui va consacrer beaucoup de temps à l'ensemble de ses activités d'enseignant (charges de cours, corrections, élaboration de nouveaux cours, permanences, responsabilités de diplôme ou d'année, etc.) ainsi qu'aux autres activités collectives (réunions de département ou d'UFR, réunions pédagogiques, élaboration de nouvelles maquettes, etc.). Même si ce n'est pas automatique, ces activités représentent autant de temps qu'il ne sera pas possible de consacrer à des publications individuelles valorisantes.
\end{abstract}

HR : On a pris au CNU, mais je pense que c'est toujours le cas aujourd'hui, les mêmes critères que pour les évaluations des équipes de recherche par l'AERES (devenue HCERES), à savoir qu'un enseignant-chercheur qu'à l'époque on disait « publiant » et qu'aujourd'hui on dit " produisant », c'est quelqu'un qui a une " production » tous les deux ans. Un chercheur CNRS, qui est chercheur à plein temps, il lui faut une publication par an. Comme nous, les enseignants-chercheurs, nous sommes chercheurs à mi-temps, c'est seulement une publication tous les deux ans. Autrement dit, c'était Michel Bussi qui l'avait écrit et je pense qu'il avait tout à fait raison, il disait : " pour être professeur il faut avoir de l'ordre d'une dizaine de publications ». Cela avait choqué parce que ça ne paraissait pas beaucoup. Dans la pratique si tu en as une tous les deux ans et que tu en as 10, tu peux avoir 20 ans de carrière derrière toi et du point de vue du CNRS tu as rempli tes obligations. Un article c'est de l'ordre d'une dizaine de pages, un chapitre d'ouvrage c'est pas forcément beaucoup plus qu'une dizaine de pages, donc être capable d'écrire une dizaine de pages de science tous les deux ans, ça ne nous paraissait pas un objet insurmontable. Il ne nous paraissait pas aberrant que quelqu'un de très investi dans des tas de choses, qui prend du temps à des corrections de copies, à des tâches pédagogiques, de montage de maquette, etc., doit quand même être capable de publier un papier tous les deux ans. Et très concrètement, quand on consulte les dossiers, on s'aperçoit que beaucoup 
de collègues font 3-4 publications par an, qui sont plutôt pas mauvaises en fait. Et certains qui font deux publications par an sont aussi investis dans une direction d'UFR, un conseil de l'université ou du PRES, de la COMUE, etc.

JG-MM-FR : Oui cette manière de voir peut s'appliquer pour des qualifications aux fonctions de maître de conférences ou de professeurs, qui ne sont pas des concours et où le nombre de qualifiés n'est pas limité, mais dès qu'il s'agit des primes (PEDR), des promotions (changements d'échelon, passages en "hors classe» pour les MCF, en "classe exceptionnelle » pour les professeurs), avec des promotions par concours et un nombre de places ridiculement bas, il y a une mise en concurrence directe beaucoup plus élevée, puisqu'il y a très peu de supports. Les collègues investis dans l'enseignement ne peuvent qu'être pénalisés par rapport à ceux qui privilégient la recherche et les publications, et qui seront d'autant plus enclins à le faire que cela favorisera leur carrière...

HR: Alors pour les PEDR, il y a quatre rubriques 嘔 publications, encadrement doctoral et scientifique, rayonnement scientifique et responsabilités scientifiques [50 auxquels s'ajoutent d'autres activités et responsabilités où l'on retrouve l'enseignement. Il est vrai cela dit qu'il y a des collègues qui ont des listes de publications ahurissantes! Mais il y avait des débats très complexes pour savoir comment les évaluer, par exemple pour savoir si une publication signée en premier ou deuxième auteur équivaut à une signature en quatrième ou cinquième auteur. L'une de nos collègues considérait qu'en géographie physique, la moindre petite contribution justifiait de mettre son nom et que le CNU devait compter un article signé par dix auteurs comme équivalent à un dixième d'article. Et d'autres qui lui répondaient qu'une manipulation sur le terrain avec du matériel lourd par exemple exigeait d'être plusieurs personnes et que cela justifiait de cosigner la publication qui en rendait compte même si chacun n'avait pas écrit le même nombre de lignes.

JG-MM-FR : Sachant que la pratique de co-signature la plus répandue en SHS est d'indiquer les auteurs par ordre alphabétique tout simplement, ce qui signifie qu'il n'y a pas de "rang » ou que le «rang » éventuel n'est pas toujours visible... A la limite, c'est uniquement quand l'ordre alphabétique n'est pas respecté que l'on peut faire l'hypothèse d'une volonté de hiérarchiser les auteurs.

HR : C'est très juste. Il y a des collègues qui mettent systématiquement l'ordre alphabétique, mais sans le faire commencer par le A, et ils changent les accents pour ne pas être reconnus par les robots qui servent aux bibliométries! Il y a d'autres personnes qui nous ont dit, et je pense que c'est un argument qui tout à fait recevable, que lorsqu'ils publient avec un doctorant, "c'est son nom qui doit être placé en premier ». Et ça, moi c'est ce que je fais.

JG-MM-FR : Au nom du fait qu'ils en ont plus besoin?

HR: Oui ! On voit qu'il y a plusieurs stratégies possibles, et je ne sais pas si l'une est meilleure que les autres.

JG-MM-FR : Oui mais si les stratégies ne sont pas les mêmes, comment les décrypter? Et le plus important est qu'une évaluation fondée uniquement ou principalement sur les publications ne peut que pousser à ces pratiques de co-signature plus ou moins stratégiques et artificielles, sans que l'on puisse, là non plus, vérifier ce qu'il en est... Autrement dit, il ne faudrait pas confondre capacité à publier, c'est-à-dire à signer un article publié, et capacité à rédiger un article, sans parler de l'activité de recherche. Cet accent mis sur les publications a d'autres effets qui sont importants. Même si l'on peut effectivement considérer que, quand on est enseignant-chercheur, une publication tous les deux ans n'est pas en théorie un objectif très difficile à atteindre, cette obsession 媐 le fameux « publish or

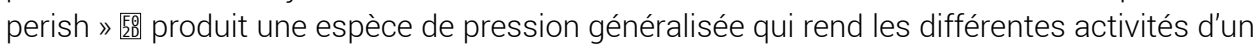
$\mathrm{E}-\mathrm{C}$ concurrentes et contradictoires les unes avec les autres. Or, on ne peut que constater 
que lorsqu'on essaie de faire le métier d'enseignant un peu correctement, on n'est pas à mitemps en fait : on est à 8 ou 9 dixièmes minimum ! L'activité de recherche, quand elle existe encore, se contente des interstices. Donc pour tous ceux qui aiment leur travail et essaient de tenir à la fois l'enseignement, la recherche, le collectif, etc., ce n'est pas un «temps plein », c'est un «temps double»: ce sont les soirées, les week-ends et les vacances qui y passent, autrement dit l'ensemble du temps censément « hors travail » qui est colonisé !

HR : Pour avoir été à plein temps dans le secondaire, dans les classes CPPN de zones prioritaires, je ne pense pas que la charge d'enseignement à l'université soit une charge importante. J'ai vu la différence quand je suis arrivé à l'université de Rennes : les étudiants sont adorables, il n'y a pas de problème de discipline, quand ils ne comprennent pas ils posent une question, etc. Sans vouloir être provocateur, depuis que je suis à l'université, j'ai toujours trouvé parfaitement normal de travailler le soir, le week-end et pendant les vacances, pour l'université. Je n'ai pas toujours eu cinq semaines de vacances par an. Je ne dois pas être le seul dans ce cas-là.

JG-MM-FR : Mais cela pose au moins deux soucis. D'une part, cette situation fait que l'un des critères qui peut jouer dans les écarts entre dossiers, un critère invisible et pourtant déterminant, c'est par exemple l'existence d'une famille, d'enfants plus ou moins nombreux et demandant plus ou moins de disponibilité. II existe clairement des inégalités dans les capacités à coloniser le hors travail. Et quand on se résout à le faire, c'est à quel prix !

HR : Oui vous avez tout à fait raison, sauf que cela, on ne peut pas demander aux gens de l'indiquer dans leur dossier. Nous l'avons pris en compte au CNU quand c'était indiqué, à l'image d'une candidate qui avait précisé qu'elle avait une enfant en situation de handicap.

JG-MM-FR : Et d'autre part, comme il y a une mise en concurrence généralisée avec une pression morale et institutionnelle, c'est...

HR : Pour les primes et les promotions oui.

JG-MM-FR: Pour les qualifications, pour les primes, pour tout. Même pour être normal! Pour être juste un collègue normal, qui ne sera pas montré du doigt. On peut penser que c'est cela qui est en train de se mettre en place avec le New Public Management et ce mélange de relocalisation et de bureaucratisation provoqué par la LRU. Or, même quand on colonise le hors travail, on ne peut pas tout faire aussi bien qu'on le voudrait. Au final, on peut comprendre que certains collègues belges en appellent à la « désexcellence » et à la "slow science» comme seul moyen de travailler correctement. Ils ont repris comme slogan un graff qu'ils ont repéré quelque part : « Vite, beaucoup, mal ».

Le collectif informel comme solution... mais comme solution locale et contingente

HR: Oui je suis d'accord sur le fond. Mais il faut nuancer le constat selon les conditions de chaque université. Il se trouve qu'à l'université de Rennes, nous nous partageons le travail: pour un certain nombre de cours, nous sommes plusieurs intervenants, nous les préparons à l'avance, nous nous les donnons les uns aux autres, donc les cours sont transparents entre collègues. Cela veut dire qu'il y a un rapport de confiance. Ensuite on fait évaluer les cours par les étudiants, sérieusement, c'est-à-dire que l'on tient compte de ce qu'ils disent. Et les étudiants jouent le jeu, c'est-à-dire qu'ils pointent ce qui est clair, ce qui ne l'est pas, ce qu'il faudrait réexpliquer différemment et ainsi de suite. C'est très enrichissant quand il y a un rapport de confiance. Les étudiants ne sont pas là pour chercher la petite bête ou au contraire pour nous dire que tout est génial en espérant une bonne note... Il y a un vrai dialogue, et à partir de ce moment-là il est possible de construire une activité d'enseignement à plusieurs. Ça marche particulièrement bien en licence professionnelle et en master géophysique, et au final cela représente moins de charge 
de travail personnelle. En quelque sorte on se partage le stress, le travail, et on s'aperçoit très vite qu'à force de discuter avec des collègues, il y a des possibilités de réfléchir et d'écrire un article sur tel ou tel sujet. Et là où ça marche le mieux, c'est pour les cours de préparation au capes et à l'agrégation. Parce qu'il n'y a personne qui maîtrise chaque année la totalité des questions, ce n'est pas possible. Donc quand il y a une nouvelle question qui apparait, ceux qui sont volontaires - on est une petite dizaine dans le département à être ainsi volontaires chaque année dans le département - se lancent et on construit le cours comme si on construisait un livre où chacun aurait un chapitre. Cela permet aussi d'intervertir nos séances si l'un d'entre nous a une indisponibilité de dernière minute. Et du point de vue intellectuel, c'est très stimulant de discuter entre collègues sur la façon de présenter tel ou tel point : « qu'est-ce que tu vas prendre comme exemple ? Est-ce que tu fais une figure? Est-ce que tu fais un croquis au tableau? Qu'est-ce que tu fais comme type d'exercice?» Etc. Donc chacun apprend en termes de pédagogie. Et puis quand quelqu'un a un doute, les collègues sont là pour aider, à la manière de referees pour un article. Il faut avoir confiance les uns dans les autres. Avec les collègues historiens et avec les collègues de l'ESPE, on organise cinq ou six réunions par an qui durent chacune une après-midi. Ce sont des réunions denses, fatigantes, mais à la fin tout le monde gagne du temps.

JG-MM-FR : Mais cela signifie que ces possibilités de réflexion sur la pédagogie, de travail collectif, etc., dépendent de l'individu, de la configuration locale de son département et de son université. II s'agit surtout d'une logique informelle d'autoformation qui est dépendante des relations avec les collègues. Tout fonctionnant à l'initiative informelle, cela ne peut prendre que s'il y a de la confiance entre collègues. Autrement dit, c'est totalement contingent. Et comme c'est contingent, comment faire quand les départements sont trop grands, quand c'est trop anonyme, ou bien quand il y a des clans, des déchirements... ? Ou comment faire pour que cela soit moins contingent? Pourquoi ça ne serait pas un peu plus officialisé ? Les E-C n'ont même pas de formation pédagogique. Celles et ceux qui ont été « moniteurs » avaient des cours (CIES) mais qui semblaient décriés ou inutiles.

HR : La formation que j'ai eue après l'agrégation pour enseigner dans le secondaire était de la foutaise intégrale. Les universités, au niveau des écoles doctorales, doivent mettre en place des formations pour les doctorants, mais elles doivent être communes à toutes les disciplines, donc même avec toute la meilleure volonté du monde, ce n'est pas très efficace. Le seul cours qui fonctionne à peu près bien, selon ce que disent certains ATER qui l'ont suivi, ce sont les cours de psychologie des étudiants : cela permet de mieux connaître leur public, leur manière de réagir, le temps qu'ils ont pour travailler, etc. Mais théoriquement, la formation des futurs E-C, c'est surtout le travail du directeur de thèse vis-à-vis du doctorant. C'est pour cela que je pense qu'on ne peut pas encadrer 15 thèses en même temps. Le directeur doit trouver un sujet qui est faisable en trois ans, parce qu'après il n'y a plus de financement, et ensuite il doit aider le thésard à faire un cours magistral, un TD, etc.

JG-MM-FR: On peut aussi considérer que cette formation à l'enseignement devrait être optionnelle parce qu'il y a différentes manières de se lancer dans une thèse et ce n'est pas forcément pour devenir enseignant-chercheur. Une thèse est, par définition, un apprentissage de la recherche, du métier de chercheur, pas d'enseignant.

Dans le secondaire, les enseignants ne sont pas évalués par leurs pairs, mais par des inspecteurs, dépendants d'une administration. L'inspecteur est censé être dépositaire d'un savoir disciplinaire et pédagogique. À l'université il n'y a pas ça du tout. II y a des contextes où le travail collectif marche, et d'autres où ça ne marche pas du tout. La section 23 du CNU a organisé récemment une séance de discussions sur le «suivi de carrière » et 
certains collègues ont dit que ce dispositif pourrait être une bonne chose pour évaluer la pédagogie parce qu'elle n'est évaluée nulle part, ou parce que même si cela fonctionne bien à l'échelle locale, il pourrait être intéressant d'avoir un regard extérieur, différent. Est-ce que I'on peut imaginer que le CNU se tourne plus du côté de l'enseignement?

HR : Je ne pense pas que ça soit le rôle du CNU. Enfin, ça pourrait être le rôle du CNU en tant qu'instance nationale. Mais je pense que pour que ça soit efficace, le meilleur moyen c'est qu'un collègue vienne faire cours avec toi et qu'après vous discutiez. Un collègue qui peut te faire une critique sans que ce soit quelque chose de désobligeant. Quelqu'un avec qui tu t'entends bien. Pour éviter qu'il y ait cette idée de jugement, qui peut être un peu agaçante, la bonne solution c'est d'aller faire un TD de terrain avec des collègues. Tu prends les étudiants pendant 8 jours et tu les fais travailler sur des problématiques très variées et le soir on fait une petite heure de discussion où chaque enseignant fait une présentation d'une demi-heure suivie d'un débat. Cela permet de voir comment les collègues parlent, et ça c'est une pure merveille. A Rennes, cela fait des années qu'on fait ça en master (pour des questions d'effectifs) et les étudiants adorent ça. Ils apprennent énormément et en même temps ça a beaucoup aidé les enseignants à améliorer leurs pratiques pédagogiques.

JG-MM-FR : Donc tu penses que le CNU ne pourrait pas le faire?

$\mathrm{HR}$ : Ça serait une excellente idée sur le plan théorique mais je ne vois pas comment la mettre en place matériellement. Le CNU pourrait éventuellement intervenir sur des sortes d'écoles thématiques. Ce sont des dispositifs qui existent pour les équipes CNRS. Il faut que ça soit entre collègues : chacun va expliquer sa compétence et va écouter la compétence du collègue, donc chacun est à la fois en situation d'être étudiant et d'être professeur. On est entre pairs. C'est du dialogue qui marche, sans enjeu hiérarchique, sans promotion à la clé. Une sorte de formation continue. Ce ne serait pas normatif, et encore une fois sur la base du volontariat.

\section{NOTES}

1. Site Internet du $\mathrm{CNU}$, recommandations pour la qualification aux fonctions de professeur des universités, consulté au moment de l'entretien, en mai 2016. Il faut noter que ces éléments ont aujourd'hui disparu.

INDEX

Thèmes : Carnets de débats 